\title{
MOLECULAR WEIGHTS OF LIQUIDS, III.
}

BY CLARENCE L. SPEYERS.

Received December 17, 1898 .

$A$ SHORT time ago, I published two papers' on this subject, A using the equation

$$
\frac{n_{1}}{n_{1}+n_{2}}=\frac{p-p^{\prime}}{p}
$$

to determine the molecular weights of liquids in homogeneous liquid mixtures. Recently some more measurements of vaporpressures of solutions have been published ${ }^{2}$ to which measurements this equation can be applied.

Using the notation and arrangements of the preceding paper, we get the following two tables:

\begin{tabular}{|c|c|c|c|c|c|c|c|}
\hline \multicolumn{8}{|c|}{$\mathrm{CCl}_{4}$ AxD $\mathrm{C}_{6} \mathrm{H}_{6}\left(50^{\circ}\right)$} \\
\hline$\frac{\dot{m}}{\varrho}$ & $\frac{\dot{\infty}}{0}$ & & & $\bar{y}$ & $=$ & $\sum^{\infty}$ & 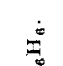 \\
\hline$\dot{\Xi}$ & $\dot{\dot{g}}$ & $\Xi$ & $\Xi^{\infty}$ & 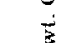 & $=$ & $\dot{3}$ & \\
\hline 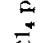 & $=$ & $0 \pi$ & $\hat{\sigma}$ & $=$ & $\Xi$ & $\stackrel{3}{i}$ & $\Sigma$ \\
\hline$\overline{8}$ & 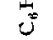 & 象 & 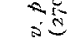 & $\frac{\mathscr{g}}{\mathrm{L}}$ & $ミ$ & $\stackrel{8}{z}$ & $\Sigma$ \\
\hline IO & 90 & 34.0 & 242.4 & I 48.4 & 0.97 & 81.2 & I.O \\
\hline 20 & 80 & 67.0 & 215.0 & I 49.3 & 0.97 & 85.7 & I.I \\
\hline 30 & $7^{\circ}$ & 98.2 & 190.0 & I 55.7 & I.O & 84.0 & I.I \\
\hline 40 & 60 & I 28.6 & 166.0 & 162.9 & I.I & 82.5 & I.I \\
\hline 50 & 50 & I 57.4 & 140.0 & I64.9 & I.I & 79.9 & I.O \\
\hline 60 & 40 & 187.5 & I I 3.0 & I65.4 & I.I & 79.0 & I.O \\
\hline 70 & 30 & 217.0 & 87.0 & I 70.0 & I.I & 77.2 & I.O \\
\hline 80 & 20 & 247.0 & 60.0 & 175.2 & I.I & $75 \cdot 3$ & 0.96 \\
\hline 90 & IO & 279.0 & 30.6 & $17^{6.3}$ & I.I & 75.8 & 0.97 \\
\hline \multicolumn{8}{|c|}{$\mathrm{CCl}_{4}$ AND $\mathrm{C}_{7} \mathrm{H}_{3}\left(5^{\circ}\right)}$. \\
\hline$\frac{n}{0}$ & $\frac{\dot{3}}{0}$ & & & 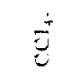 & $\vec{j}$ & 5 & $\equiv$ \\
\hline$\stackrel{\vec{u}}{g}$ & 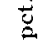 & $\dot{\tilde{u}} \dot{\partial}$ & $=$ & 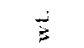 & $=$ & 3 & $=$ \\
\hline$\ddot{0}$ & \pm & $\therefore \varrho$ & $<\hat{0}$ & $\bar{z}$ & & $\sigma$ & $\Sigma$ \\
\hline & $\tilde{j}$ & $\dot{2} \cong$ & is & 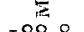 & $\Xi$ & $\Sigma$ & ב \\
\hline Io & 90 & 27.2 & $85 \cdot 3$ & I 88.8 & I.2 & 79.4 & 0.86 \\
\hline 20 & 80 & 54.0 & 77.4 & 190.2 & I.2 & 77.4 & 0.84 \\
\hline $3^{\circ}$ & 70 & 82.4 & $69 . I$ & 190.0 & I. 2 & 77.4 & 0.84 \\
\hline 40 & 60 & I I 1.4 & 60.8 & $I 88.4$ & I. 2 & 77.0 & 0.84 \\
\hline 50 & 50 & I 43.0 & 52.0 & I 94.5 & I.3 & $7^{8} \cdot 3$ & 0.85 \\
\hline 60 & 40 & \pm 75.4 & 42.7 & 195.3 & I. 3 & 73.9 & 0.80 \\
\hline 70 & 30 & 208.2 & 33.0 & 195.9 & I.3 & 79.9 & 0.87 \\
\hline $8 c$ & 20 & 240.0 & 22.7 & I98.I & $\mathrm{I} \cdot 3$ & 77.9 & 0.85 \\
\hline 90 & IO & 274.0 & II.8 & 200.6 & I. 3 & 80.3 & 0.87 \\
\hline
\end{tabular}

The plots corresponding to these tables are given in Fig. I.

1 Jour. phys. Chem., 2, 347, 362 (1898).

2 Lehfeldt : Phys. Mag , 46,42 (7898). 


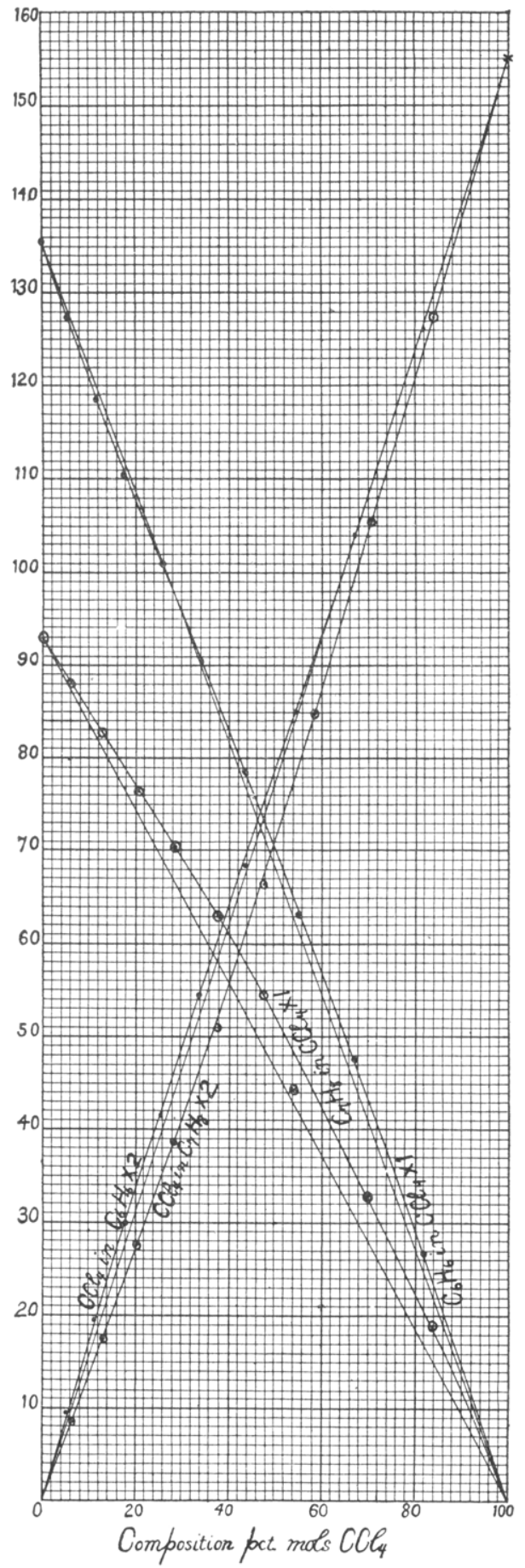

Fig. I. 
The vapor-pressure plot of benzene indicates a slight association of carbon tetrachloride when the mixture is concentrated with respect to carbon tetrachloride, but a slight dissociation when the mixture is dilute with respect to it. In view of the nature of carbon tetrachloride, I think we should take this to mean that carbon tetrachloride has a normal molecular weight when mixed with benzene.

The vapor-pressure plot of carbon tetrachloride indicates a slight dissociation of benzene when the mixture is concentrated with respect to benzene, but not enough to show an association of the benzene molecules. The very peculiar plot given by Linebarger' is not found here.

The vapor-pressure plot of toluene indicates an association of carbon tetrachloride, but as there was no indication of association in the experiments of Linebarger, I think we are to conclude that carbon tetrachloride has a normal molecular weight in toluene.

The vapor-pressure plot of carbon tetrachloride would indicate a slight dissociation of toluene, but this is hardly admissible, as here likewise we must conclude that toluene has a normal molecular weight in carbon tetrachloride, particularly since the experiments of Linebarger point to the same conclusion.

$$
\mathrm{C}_{2} \mathrm{H}_{5} \mathrm{OH} \text { AND } \mathrm{C}_{6} \mathrm{H}_{6}\left(50^{\circ}\right) \text {. }
$$

\begin{tabular}{|c|c|c|c|c|c|c|}
\hline 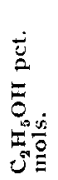 & 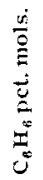 & 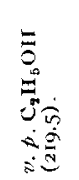 & 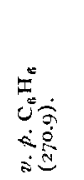 & 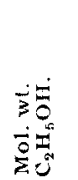 & $\begin{array}{l} \pm \\
0 \\
= \\
0 \\
\vdots \\
\vdots \\
\vdots\end{array}$ & $\begin{array}{l}\dot{0} \\
= \\
\dot{0} \\
\dot{3} \\
\dot{3} \\
\dot{0}\end{array}$ \\
\hline IO & 90 & I 10.0 & 264.4 & 205.1 & 4.4 & 705.0 \\
\hline 20 & 80 & $I 35.0$ & 256.6 & 206.3 & 4.5 & $49^{8} .0$ \\
\hline 30 & 70 & I5I.0 & 248.0 & $2 \mathrm{I}_{3} .5$ & 4.6 & 401.0 \\
\hline 40 & 60 & 164.0 & 237.4 & 217.3 & 4.7 & 346.0 \\
\hline $5^{\circ}$ & 50 & $\mathrm{I} 75.0$ & 225.8 & 230.6 & 5.0 & 307.0 \\
\hline 60 & 40 & 185,2 & 207.6 & 226.3 & 4.9 & 281.0 \\
\hline 70 & 30 & I94.0 & $I 83,0$ & 223.5 & 4.9 & 254.0 \\
\hline 80 & 20 & 203.2 & I 46.0 & 215.2 & 4.7 & 240.0 \\
\hline go & Io & $2 \mathrm{II} .4$ & 96.4 & 228.8 & 5.0 & 226.0 \\
\hline
\end{tabular}

1 This Journal, $17,6 \mathrm{r}_{5}, 690$ (1895); Speyers : J.phys. Chem., 2, 347 (1898). 


$$
\mathrm{C}_{2} \mathrm{H}_{5} \mathrm{OH} \text { AND } \mathrm{C}_{7} \mathrm{H}_{8} \text {. }
$$

\begin{tabular}{|c|c|c|c|c|c|c|c|}
\hline 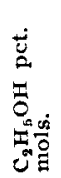 & 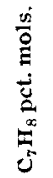 & 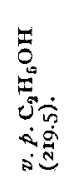 & $\begin{array}{l}\underbrace{\infty} \\
\dot{5} \cdot \dot{0} \\
\dot{0}\end{array}$ & 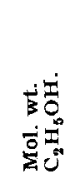 & $\begin{array}{l}5 \\
0 \\
5 \\
5 \\
5 \\
\Sigma \\
\Sigma\end{array}$ & $\begin{array}{l}\dot{a} \\
\dot{v} \\
\dot{v} \\
\dot{\vec{b}} \\
\dot{\vec{a}}\end{array}$ & $\begin{array}{l}\dot{0} \\
0 \\
\vdots \\
\vdots \\
\vdots\end{array}$ \\
\hline IO & 90 & I IO.O & 86.8 & 71.6 & 1.6 & 831.0 & 9.0 \\
\hline 20 & 80 & I 35.0 & 83.0 & 95.5 & $2 . I$ & 588.0 & 6.4 \\
\hline 30 & 70 & I50.6 & 80.5 & 127.0 & 2.8 & 469.0 & $5 . I$ \\
\hline 40 & 60 & $I 62.2$ & 77.8 & I 57.0 & 3.4 & 390.5 & 4.2 \\
\hline 50 & 50 & I69.6 & 75.2 & 194.3 & 4.2 & 312.7 & 3.4 \\
\hline 60 & 40 & 174.4 & 71.9 & $235 . I$ & $5 . I$ & $237 \cdot \mathrm{I}$ & 2.6 \\
\hline 70 & 30 & I 79.0 & 66.8 & 273.7 & 5.9 & I 74.3 & I.9 \\
\hline 80 & 20 & I 87.8 & $57 \cdot 3$ & 295.4 & 6.4 & 136.2 & I.5 \\
\hline 90 & 10 & 203.6 & $3^{6.9}$ & 272.2 & 5.9 & 130.9 & I. 4 \\
\hline
\end{tabular}

The plots corresponding to these two tables are given in Fig. 2. We see from the vapor-pressure of alcohol that benzene and toluene are polymerized to a remarkable degree in alcohol, and very equally so from a concentration of roo per cent. grammolecules of benzene or toluene down to about sixty per cent. gram-molecules benzene or toluene, but from about sixty per cent. gram-molecules of these two liquids down, they are not equally polymerized in alcohol. The vapor-pressure plot of alcohol with toluene runs so as to indicate a normal molecular weight for toluene in alcohol when the mixture is dilute with respect to toluene, but the vapor-pressure plot of alcohol with benzene does not indicate a normal molecular weight for benzene at any dilution.

We see from the vapor-pressure plot of toluene that alcohol is highly polymerized in toluene, but when the concentration of the toluene is high, the molecular weight of the alcohol drops towards a normal value. This corresponds to the bebavior of toluene in alcohol.

The vapor-pressure plot of benzene, however, indicates that alcohol is highly polymerized in benzene at all concentrations. This corresponds to the behavior of benzene in alcohol. On the other hand, there is a possibility that alcohol has a normal molecular weight in benzene when the mixture is dilute with respect to alcohol, for there are two observations of the vaporpressure of benzene at about $125 \mathrm{~mm}$. mercury, which lie very 


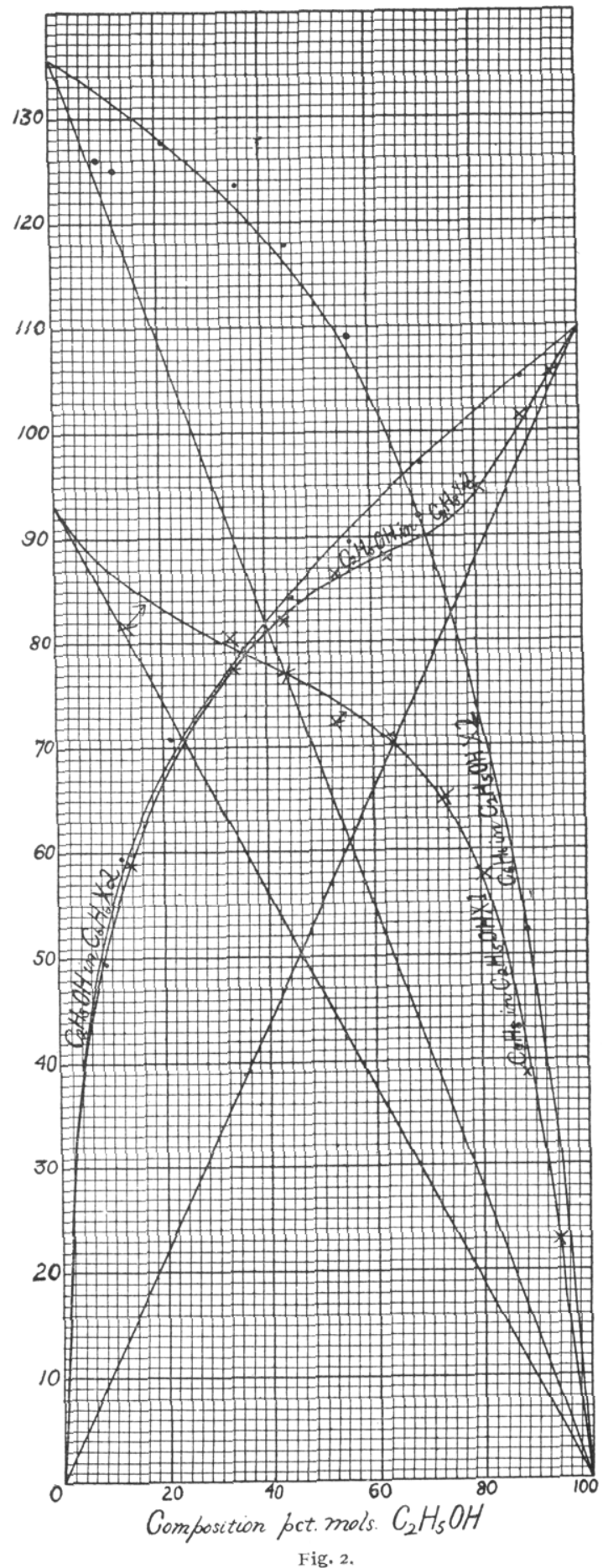


close to the diagonal. If these observations are correct, then alcohol would have nearly normal molecular weights at the concentrations corresponding to these observations, but these observations are so different from the other ones that I thought it best to draw the plot as I did.

RUtgers College, December 16,1898 .

\section{A NEW FILTERING MEDIUM. ${ }^{1}$}

By GEo. WM. SARgeNt AND JNo. KIRK FAUSt.

Received November 23, 1898 .

COR the removal of manganese dioxide from the nitric acid in which solution it has been precipitated by potassium chlorate, a glass tube similar to that used for carbon determinations, is recommended. The bottom of this tube is covered first with a small piece of glass wool, then with asbestos. This we used for some time, but were frequently troubled by the precipitate running through, unless a very thick bed of asbestos was made. In this case, the time consumed in filtering was very considerable, due to clogging of the filter by potassium chloridefrom the large amount of potassium chlorate necessary to oxidize the chromium which was present in considerable quantities in the steels dealt with.

After some experimenting, we adopted the following as giving the most satisfactory results: A carbon filtering tube with a stem five inches long and a body three and one-half inches long and one and one-fourth inches in diameter, is filled one-fourth inch with pewter sand, a small piece of glass wool being used to retain the sand; over this a thin layer of asbestos is formed by pouring in the asbestos shaken up with water and sucking the bed dry. A little hot dilute nitric acid is poured back and forth through the tube several times, when it is ready for use.

When a heavy precipitate is encountered, the bulb from a syringe is used to force air into the tube and drive the liquid through, care being taken to keep the bulb compressed until removed from the tube.

We have found that the quickest and best way to wash the manganese dioxide consists in allowing the nitric acid to run

1 Read at the New York meeting of the American Chemical Society, December 28 , I 898 . 